Mens

revue d'histoire intellectuelle de l'Amérique française

MENS

\title{
Sur Nationalisme et religion (1936) de Louis Lachance, o. p.
}

\section{Yves Bégin}

Volume 2, numéro 2, printemps 2002

URI : https://id.erudit.org/iderudit/1024608ar

DOI : https://doi.org/10.7202/1024608ar

Aller au sommaire du numéro

Éditeur(s)

Centre de recherche en civilisation canadienne-française

ISSN

1492-8647 (imprimé)

1927-9299 (numérique)

Découvrir la revue

Citer cet article

Bégin, Y. (2002). Sur Nationalisme et religion (1936) de Louis Lachance, o. p. Mens, 2(2), 155-192. https://doi.org/10.7202/1024608ar

\section{Résumé de l'article}

$\mathrm{Au}$ Québec comme ailleurs, et pour diverses raisons, les rapports entre le nationalisme et la religion ne sont pas toujours allés de soi. Ici, les liens étroits entretenus pendant longtemps entre l'Église catholique et les nationalistes canadiens-français (on parle souvent de clérico-nationalisme) n’ont pas empêché que soit posée la question suivante : peut-on être à la fois nationaliste et catholique ? Certains événements comme la mise à l'Index de L'Action française de Charles Maurras en France en 1926 ainsi que la crise sentinelliste, qui a déchiré les Franco-Américains en 1928, ont eu des répercussions ici et posé ce problème avec plus d'acuité. En 1936, un jeune prêtre et philosophe dominicain canadien-français, Louis Lachance, a tenté d'y apporter une réponse dans un essai intitulé Nationalisme et religion. Pour Lachance, il n’y a de nationalisme légitime que lorsque subordonné et encadré par la religion. Cet article expose les thèses contenues dans ce livre et rappelle entre autres qu'elles ont été reçues favorablement par l'un des critiques les plus véhéments du nationalisme canadien-français de l'époque, Jean-Charles Harvey.
Ce document est protégé par la loi sur le droit d'auteur. L'utilisation des services d’Érudit (y compris la reproduction) est assujettie à sa politique d'utilisation que vous pouvez consulter en ligne.

https://apropos.erudit.org/fr/usagers/politique-dutilisation/ 


\title{
SUR NATIONALISME ET RELIGION (1936) DE LOUIS LACHANCE, O.P.
}

\author{
Yves Bégin \\ Département d'histoire \\ Université Laval
}

\section{RÉSUMÉ}

Au Québec comme ailleurs, et pour diverses raisons, les rapports entre le nationalisme et la religion ne sont pas toujours allés de soi. Ici, les liens étroits entretenus pendants longtemps entre l'Église catholique et les nationalistes canadiens-français (on parle souvent de clérico-nationalisme) n'ont pas empêché que soit posée la question suivante : peut-on être à la fois nationaliste et catholique? Certains événements comme la mise à l'Index de L'Action franfaise de Charles Maurras en France en 1926 ainsi que la crise sentinelliste, qui a déchiré les Franco-Américains en 1928, ont eu des répercussions ici et posé ce problème avec plus d'acuité. En 1936, un jeune prêtre et philosophe dominicain canadien-français, Louis Lachance, a tenté d'y apporter une réponse dans un essai intitulé Nationalisme et religion. Pour Lachance, il n'y a de nationalisme légitime que lorsque subordonné et encadré par la religion. Cet article expose les thèses contenues dans ce livre et rappelle entre autres qu'elles ont été reçues favorablement par l'un des critiques les plus véhéments du nationalisme canadien-français de l'époque, Jean-Charles Harvey.

\section{ABSTRACT}

For a variety of reasons, and despite the close ties which have bistorically linked the Roman Catholic Church to Quebec's nationalist movement (many authors even speak of "clerical 
nationalism" to describe traditional French Canadian nationalism), the relationship between the two groups has at times been strained. These tensions were not unique to French Canada. Indeed, in the wake of certain events, most notably New England's notorious Sentinelle Affair and Pope Pius XI's 1926 condemnation of French monarchist Charles Maurras' Action française - both of which shook French Canadian nationalism to the core -, Catbolic thinkers the world over pondered the following question: Could one be at once a nationalist and a Roman Catholic? In 1936 a young French Canadian priest and Dominican philosopher, Louis Lachance, sought to answer this fundamental question in an essay titled Nationalisme et religion. He believed that nationalism could only be legitimate when it was framed by and subordinated to religion. This article explores the ideas contained in this key text. It also examines the favourable reception that Jean-Charles Harvey, one of French Canadian nationalism's most vebement and persistent critics, gave to Nationalisme et religion.

S'il est vrai que notre histoire intellectuelle suscite de plus en plus l'intérêt des chercheurs et du public lecteur, il y a malheureusement tout un pan de cette histoire qui a été trop peu étudié : la philosophie. Quelques pionniers s'y sont bien intéressés, mais on ne peut certes pas parler de la philosophie comme d'un champ de recherche historique véritablement constitué au Québec. Une recherche non exhaustive nous révèle que pour une période d'environ trente ans, à peine une dizaine d'ouvrages sont parus à sont sujet $^{1}$. Historiquement, le Canada français n'a peut-être pas produit de figures majeures à ce chapitre, mais il reste que nous avons eu nos philosophes et que la philosophie a toujours été une composante majeure de l'enseignement au Québec. Le manque d'intérêt pour la philosophie d'ici peutil s'expliquer par le fait que plusieurs de nos philosophes furent surtout des théologiens, et que la pensée thomiste, 
qui a servi d'objet et d'inspiration à ceux-ci plus que toute autre, a été disqualifiée et n'intéresse aujourd'hui plus personne ou presque? C'est notre avis. Ainsi tombées en désuétude, la théologie et la philosophie religieuse auront entraîné avec elles la mémoire de leurs artisans.

Parmi ces artisans oubliés se trouve le philosophe et théologien dominicain Louis Lachance. Né le 18 février 1899 à Saint-Joachim-de-Montmorency (village qui aura aussi donné au Québec le sociologue Fernand Dumont), Joseph-Henri Lachance a consacré sa vie à la philosophie et à son enseignement. Après avoir complété ses études au Petit Séminaire de Québec, il revêt à vingt et un ans l'habit dominicain au noviciat de Saint-Hyacinthe et prend le nom de Frère Louis. Après de brillantes études au Couvent d'Ottawa durant lesquelles il est ordonné prêtre et décroche le titre de Lecteur en théologie, Lachance est appelé à enseigner la philosophie par ses supérieurs, qui l'envoient bientôt à Rome pour qu'il y complète son agrégation (maîtrise). De retour au Canada en 1931, il enseigne durant cinq ans la philosophie à Ottawa et collabore à la fondation de l'Institut d'études médiévales. Au cours de cette décennie fort active, Lachance publie pas moins de cinq ouvrages ${ }^{2}$ dont le troisième, Où vont nos vies?, lui mérite en 1934 le prix d'Action intellectuelle de l'Action catholique de la jeunesse canadienne-française. Le jeune philosophe attire alors de plus en plus l'attention, notamment celle de Lionel Groulx ${ }^{3}$, mais aussi celle du chanoine Philippe-Servule Desranleau, futur évêque de Sherbrooke (1941-1952). En 1938, alors qu'il revient d'un second séjour à Rome où il avait enseigné la philosophie sociale et défendu sa thèse de maîtrise en théologie, Lachance reçoit l'invitation de Desranleau à faire partie des fondateurs du Grand Séminaire des Saints-Apôtres dont on vient d'entreprendre la construction à Sher- 
brooke. Desranleau voulait s'assurer, en conformité avec les directives romaines, que les futurs professeurs du Séminaire fassent tous une propédeutique en philosophie avant leur première année d'enseignement. C'est en Louis Lachance qu'il a trouvé le maître capable de s'acquitter de cette tâche. Les cinq années qu'il passe ensuite à Sherbrooke lui permettent de publier un sixième ouvrage ${ }^{4} e t$ sont couronnées en 1944 d'un événement important, celui de son accession au titre de Maitre en sacrée théologie, le plus haut titre universitaire dans l'Ordre des Dominicains. La même année, il est nommé professeur de philosophie morale et sociale à la Faculté de philosophie de l'Université de Montréal, où il enseignera jusqu'à son décès en 1963, et il participe à la fondation de l'Académie canadienne-française animée par Victor Barbeau. Après être allé en Espagne vers la fin des années quarante, il se rend en 1950 en Argentine et au Brésil pour y donner des cours et des conférences. Deux ans plus tard, deux honneurs lui échoient alors qu'il est nommé Supérieur du Couvent dominicain d'Outremont et qu'il accède au vice-décanat de la Faculté de philosophie, dont il deviendra finalement le doyen en 1960. C'est au cours des années cinquante qu'il publie ses trois derniers ouvrages ${ }^{5}$. À la même époque, il a aussi animé l'émissionforum L'beure dominicale à Radio-Canada, participé à la direction des Semaines sociales du Canada ainsi qu'à la fondation de la Société Richelieu d'Ottawa et enfin il s'est impliqué au sein de la Société Saint-Jean-Baptiste et de la Société de Philosophie de Montréal ${ }^{6}$. Louis Lachance est décédé en 1963.

Avec neuf ouvrages à son actif, ainsi que plusieurs articles et critiques littéraires publiés entre 1927 et 1963, l'œuvre de Lachance apparait assez imposante ${ }^{7}$. Assez imposante, du moins, pour que l'on ne songe pas nous-même 
à l'attaquer de front. Dans cet article, nous n'aborderons véritablement qu'un seul aspect de sa pensée, soit sa réflexion sur les rapports entre le nationalisme et le catholicisme.

Ces rapports ne sont pas toujours allés de soi. En Europe, au cours du $\mathrm{XIX}^{\mathrm{e}}$ siècle, plusieurs événements ont amené l'Église à condamner certaines formes de nationalisme. En France, par exemple, les idées de souveraineté populaire et de séparation de l'Église et de l'État, idées modernes, libérales et nationalistes, sapaient l'autorité de l'Église. Encore plus proche de Rome, la lutte nationaliste pour l'unification italienne fit perdre à l'Église ses possessions territoriales. Parmi les propositions condamnées par Pie IX en 1864 dans son Syllabus errorum, on ne s'étonnera donc pas de voir figurer, aux côtés du rationalisme, du libéralisme et du socialisme, l'idée accordant à la nation la liberté de décider de son destin. Plus tard, au cours du $\mathrm{XX}^{\mathrm{e}}$ siècle, Pie XI (1922 à 1939) mit à plusieurs reprises les catholiques en garde contre certaines formes de nationalisme. L'encyclique Ubi arcano, promulguée en 1922, dénonçait le nationalisme «immodéré», compris comme celui n'étant pas «réglé par la loi chrétienne» et "transgressant les règles de la justice et du droit ${ }^{8} \%$. En 1926, Rome mettait à l'Index L'Action française de Charles Maurras. Sa faute? «Ne pas subordonner sa doctrine politique à la morale chrétienne, et [...] réduire le catholicisme à une simple valeur culturelle, au rôle de gardien de l'ordre social et de l'identité nationale». En 1931, Non abbiamo bisogno condamnait l'étatisme du régime fasciste de Mussolini qui retirait à la famille et à l'Église leurs prérogatives sur l'éducation des enfants. Enfin, Mit brennender Sorge (1937) condamnait le national-socialisme allemand pour son paganisme qui le faisait ériger la race et l'État en absolus. Il est important de 
remarquer que ces condamnations concernaient des abus $\mathrm{du}$ nationalisme, et non le nationalisme en soi.

Si donc l'histoire du nationalisme et du catholicisme a pu être marquée par la méfiance et le conflit, on constate par ailleurs que certains groupes, quand ce n'est pas l'Église elle-même, les ont intégrés de façon plutôt harmonieuse. $\mathrm{Au}$ Québec, on aurait tort d'exagérer l'unanimité du clergé catholique canadien-français sur cette question, mais il semble admis que l'Église a de façon générale appuyé le nationalisme. L'Église catholique canadienne-française n'a-t-elle pas été, comme l'a affirmé le Rapport Dumont, «solidaire d'une longue lutte pour la survivance'»? Cela dit, les mises en garde sérieuses et répétées de Rome contre le nationalisme immodéré ou outrancier ne sont pas passées ici inaperçues. La mise à l'Index de L'Action française de Paris à la fin de 1926 a d'abord eu l'effet d'une bombe chez les nationalistes canadien-français, et principalement parmi ses animateurs, dont beaucoup étaient d'abord des membres du clergé. Il faut dire qu'au même moment, Henri Bourassa, qui venait d'être reçu en audience par Pie XI, revenait de Rome transformé. Depuis quelques années déjà, le chef nationaliste s'était quelque peu distancié du mouvement qu'il avait lui-même fortement contribué à relancer. Bouleversé par la mise en garde du Pape à l'égard du nationalisme outrancier, Bourassa semblait presque renier son action passée et il devint très critique face à ses compatriotes qui avaient tendance, à son avis, à confondre le national et le religieux ou qui, pire encore, semblaient placer l'appartenance nationale au-dessus de l'appartenance religieuse ${ }^{10}$. À Montréal, des soupçons s'élevèrent rapidement sur les liens entre le journal de Paris et la revue montréalaise du même nom, que dirigeait alors depuis une dizaine d'années le nouveau chef de file nationaliste, l'abbé Lionel Groulx. 
Pour éviter la confusion, L'Action française devint bientôt L'Action canadienne-française et Groulx, malgré ce qu'il laissera entendre ${ }^{11}$, restera longtemps inquiet des remises en question de la légitimité d'être à la fois nationaliste et catholique. Que dire aussi de la mise à l'Index du journal franco-américain La Sentinelle (Woonsocket, Rhode Island) et de l'excommunication des principaux leaders de l'agitation sentinelliste en $1928^{12}$ ?

Dans un tel contexte, il n'est pas étonnant que plusieurs nationalistes canadiens-français aient cherché à vérifier d'eux-mêmes si leur nationalisme n'entrait pas en conflit avec la doctrine et la morale catholiques. Louis Lachance, alors jeune religieux nationaliste, s'y emploiera de brillante façon en 1936 dans un essai intitulé Nationalisme et religion. Dix ans après l'événement, l'impact de la condamnation de 1926 est patent et l'auteur y fait d'ailleurs référence de façon explicite à quelques reprises. Soucieux de garder ses compatriotes à l'abri d'une condamnation romaine, il tente de découvrir une formule qui consacrerait la légitimité du nationalisme aux yeux de l'Église. Plus concrètement, son objectif est de vérifier si le nationalisme «comporte quelque incompatibilité» avec la religion catholique ${ }^{13}$.

Comme pour plusieurs de nos philosophes et théologiens, dont le plus connu est certainement Louis-Adolphe Pâquet ${ }^{14}$, c'est l'œuvre de saint Thomas qui est la grande inspiratrice de l'activité intellectuelle de Lachance. Quand il n'étudie pas la pensée thomiste pour elle-même, lorsqu'il s'intéresse au droit, au langage ou au nationalisme par exemple, le thomisme supporte et guide sa réflexion, avec toute la souplesse en même temps que la rigueur qui le caractérise.

Cette réflexion, Lachance désire tout particulièrement l'offrir à la jeunesse. Celle-ci, dans les années trente, est 
d'ailleurs au cœur des préoccupations de beaucoup d'intellectuels. Lachance constate avec d'autres qu'en ces années difficiles où diverses idéologies se bousculent et courtisent les insatisfaits, et devant un avenir qui leur semble bloqué, les jeunes sont particulièrement désemparés. En tant que nationaliste, il semble aussi inquiet de l'efficacité de la critique antinationaliste portant sur l'incompatibilité entre le nationalisme et la religion catholique :

On a même agité devant l'intelligence désarmée des jeunes le spectre de l'anathème : ce qui n'a pas été de nature à les rassurer. Nous croyons donc qu'il y a, à l'heure actuelle, pour tous ceux qui sont commis à la direction des âmes, un devoir impérieux, imminent, d'étudier ce problème à l'aide des principes de la raison et de la foi (p. 20).

Nous verrons plus loin qu'un de ces antinationalistes, et non le moindre, a été conquis par l'exposé de Lachance : Jean-Charles Harvey.

Même s'il ne cache pas son intérêt pour le nationalisme canadien-français, Nationalisme et religion, annonce$\mathrm{t}$-il, ne porte pas spécifiquement sur ce cas. Les principes contenus dans son essai, annonce Lachance, ont une ivaleur universelle». Qu'a-t-il à dire sur le nationalisme d'ici? De façon générale, ses compatriotes n'auraient rien de grave à se reprocher, mais ne seraient pas non plus sans faute. Il laisse ainsi entendre qu'on ne peut a priori leur donner l'absolution : "Pour ce qui est du nationalisme canadien-français, dit-il, il n'a pas encore remué mer et monde; il n'est qu'à l'état embryonnaire, [mais] nous concéderions volontiers que, bien qu'encore dans ses langes, il n'est pas immunisé contre tout défaut.» (p. 72) Un de ces défauts, selon Lachance, serait de pratiquer un nationalisme essentiellement sentimental : 
On ne saurait trouver chez nous une opinion condamnable et une conduite erronée en matière de nationalisme. Nos doctrines, lorsqu'elles ont existé, ont toujours été de la plus stricte orthodoxie. Tout ce qu'on pourrait nous reprocher, c'est de n'en avoir pas assez, c'est de ne pas nous soucier d'étayer notre action de principes, de ne pas suffisamment pratiquer l'éclairage de nos sentiments, de nos réclamations et de notre conduite (p. 16).

Si Lachance semble prêt à accepter certaines critiques, il n'entend pas écouter celles des gens qui confondent le nom et la chose. Ce n'est pas parce qu'on appelle «nationalisme» les doctrines qui sévissent en Europe et qui ont été condamnées par le Pape, soutient-il, qu'on doit condamner pour autant le nationalisme canadien-français :

Grouper ensemble et assimiler en tout point des réalités presque équivoques et parfois totalement disparates, sous prétexte qu'on les a arbitrairement coiffées du même nom, les envelopper des mêmes suspicions, du même discrédit, des mêmes condamnations, est faire preuve de mauvaise foi ou de stupidité consommée (p. 73).

Mais le nationalisme canadien-français n'est pas nécessairement sans reproche et voici ce qu'il propose à ses compatriotes : «Observons notre situation à nous et raisonnons sur les données de fait en nous éclairant d'eux [sic]; confrontons notre conduite à la règle, et s'il y a accord, marchons en toute assurance, s'il y a désaccord, corrigeonsnous.» (pp. 72-73. Nous souligons)

Nationalisme et religion est un essai qui mériterait une analyse beaucoup plus détaillée que ce que nous pouvons nous permettre de faire ici, et nous nous limiterons à exposer la synthèse des principales idées-forces contenues dans ce livre. Nous aborderons cinq thèmes fondamentaux : la 
domination de l'esprit sur la matière; le racisme comme doctrine condamnable; la nécessaire diversité des nations; la distinction entre le politique et le «national» et en dernier lieu, la compatibilité de la foi catholique et du «national». Enfin nous nous pencherons brièvement sur le cas d'un lecteur inattendu de Lachance : Jean-Charles Harvey.

\section{Les idées-force de Nationalisme et religion}

\section{a) Le nationalisme comme sentiment devant être rationalisé}

Il y a un fait de base à considérer dans l'étude de la pensée de Lachance : la prédominance qu'il accorde, en bon catholique, à l'esprit sur le corps, sur la matière. Dans un passage où il tente d'évaluer la force de l'un et de l'autre, il constate et concède en même temps que la force issue de la matière est telle que l'esprit ne peut espérer la réprimer entièrement. Il ne reste à l'esprit qu'à en faire son parti et tenter de la canaliser en la soumettant à sa volonté. Cette affirmation de «la suprématie de l'intelligence et de la foi» est fondamentale, car elle commande toute la position de Lachance au sujet du nationalisme. En effet, il faut voir qu'à l'origine du nationalisme existe un sentiment (le «sentiment national») qui, dans l'esprit du philosophe, est un sentiment naturel. Étant naturel, ajoute-t-il aussitôt, sa légitimité est incontestable : "On n'anathématise pas la nature, mais on la sauve.» (p. 69)

Lachance remarque aussi que ce qui lie concrètement les individus entre eux et donne naissance à la nation, c'est surtout un "agrément de vivre ensemble», lui-même fondé sur des affinités de tempérament (p. 87). Ce qui forme la nation, dit-il, ce qui en est l'essence, ce sont surtout les attaches sentimentales ${ }^{15}$. Mais en définitive, sur quoi ces atta- 
ches sentimentales se fondent-elles? Quelles en sont les causes réalisatrices? Aux yeux de Lachance, le sentiment national est d'abord un instinct qui «s'enracine dans la chair et le sang» (p. 57). Ainsi, l'apparition du sentiment national est en partie l'effet d'une hérédité commune, les caractères communs devenant à son avis comme le «support physiologique du sentiment national.» (p. 91) Mais il ne peut y avoir que cela : l'affirmer serait évidemment donner trop d'importance à la matière. D'ailleurs, si Lachance avait trop insisté sur l'influence de l'hérédité dans la formation du sentiment national, il n'aurait pu affirmer comme il le fait qu'il «n'est pas rare $[. .$.$] que des individus de race étrangère$ [soient] parfaitement assimilés par la nation qui les accueille et deviennent de fervents nationaux.» (p. 85) Il y a donc d'autres facteurs, plus significatifs, à considérer. Lachance en identifie trois : le facteur social, le facteur religieux et la langue. Disons quelques mots des trois.

Par facteur social, Lachance entend les traditions, les mœurs collectives et les institutions (familiales, politiques, économiques, etc.). «Par la contrainte qu'elles exercent sur les nationaux, dit-il, elles engendrent des types communs de conduite qui laissent des traces dans leurs forces motrices et qui finissent par les modifier.» (p. 99) Par facteur religieux, il entend que la religion, "par ses dogmes, sa morale, ses institutions, ses rites, [...] impose une forme à la pensée, au sentiment et à l'activité des nationaux.» (p. 100) Enfin, il considère la langue comme le facteur principal, puisqu'elle englobe tous les autres, et «leur [sert] constamment de véhicule» (p. 100). Il y aurait long à dire sur ce sujet que Lachance étudiera en profondeur quelques années plus $\operatorname{tard}^{16}$, mais nous devons nous contenter ici de l'essentiel, à savoir qu'il considère la langue comme uune fabricatrice à nulle autre pareille de mentalité commune. 
[...] Elle uniformise, ajoute-t-il, l'âme nationale.» (p. 101). Il y a donc pour Lachance des facteurs autres que strictement physiologiques et héréditaires dans la formation du sentiment national.

Le sentiment national, dans son esprit, constitue donc concrètement une prise de conscience de cette individualité propre au groupe et d'une solidarité profonde entre ses membres. De cette prise de conscience jaillit un instinct de conservation, qui s'exprime généralement en vouloir-vivre collectif ${ }^{17}$ et surtout en piété (reconnaissance, attachement respectueux) pour les causes de cette existence : Dieu, comme cause première, et les ancêtres comme causes secondes. De l'avis de Lachance, rien de bien dangereux dans tout cela. Mais il est vrai, constate-t-il, que le sentiment national, de par son origine partiellement physique, est un sentiment puissant : «il n'y a donc pas à s'étonner, dit-il, de ce que les premières manifestation [du sentiment national] prennent la tournure d'un assaut de tempérament. Le sang bouillonne sans cesse en nous et il influe constamment sur nos états psychiques.» (p. 111). Le nationalisme actuel, ditil (sans toutefois préciser s'il s'agit du nationalisme en Europe ou du nationalisme canadien-français), est justement caractérisé par l'élément passionnel (p. 110). Pourtant, cela ne le condamne pas automatiquement aux yeux de Lachance. Selon lui, en effet, les passions sont des «forces neutres», c'est-à-dire surtout qu'elles ne sont pas nécessairement mauvaises. Neutres, elles peuvent être utilisées à bon ou à mauvais escient, et c'est là qu'intervient la nécessité de la suprématie de l'esprit sur le corps : «Il y a non seulement obligation et possibilité de maitriser les passions, mais il y a grand avantage, dit-il, car une fois domestiquées, elles deviennent des énergies de haute valeur.» (p. 116) 
C'est d'ailleurs sur cette base que Lachance établit la différence entre nationalisme et patriotisme. À son avis, «le patriotisme est la forme épurée et ennoblie du nationalisme. [...] [Le premier, dit-il, est caractérisé] par l'emprise de l'élément humain, de l'élément rationnel. [Le second] par la prédominance de l'élément sentimental et passionnel ${ }^{18} \%$. Si donc le nationalisme est d'abord un sentiment puissant, mais «indifférent à être justifié ou orienté par telle ou telle philosophie» (p. 61), quelle "philosophie» doit-elle en assurer le contrôle? Bien sûr, Lachance répond qu'il s'agit de la religion catholique. Mais avant de préciser les modalités de l'expression d'un nationalisme encadré par le catholicisme, il explore une autre possibilité, par ailleurs aussitôt condamnée : le racisme.

\section{b) Le racisme, une doctrine condamnable}

L'exemple du nationalisme tel que le pratiquait l'Allemagne hitlérienne a provoqué bien des équivoques au sujet du nationalisme canadien-français, et ce jusqu'à nos jours. Mais avant le début de la guerre, avant les premières constatations des crimes horribles commis par les régimes hitlérien et mussolinien, Lachance condamnait cette forme de nationalisme.

Pour Lachance, et comme bon nombre de ses contemporains, le concept de nation décrit une réalité à la fois d'ordre culturel et biologique. Il rejette d'ailleurs l'idée d'une nation purement contractuelle :

Nous écartons aussi la conception de certains esprits pragmatiques, commercialisés, estimant tout sous l'angle de l'intérêt matériel. Il faut avoir une bien piètre idée de la profondeur de l'humain et des poussées sourdes de la race pour penser que l'unité d'intérêt suffit à engendrer l'unité nationale. À les 
entendre on croirait qu'une nation est une vaste compagnie d'assurance, une entreprise commerciale, une société de secours mutuels, une firme quelconque (p. 78-79).

Il croit fermement déceler un certain déterminisme psychologique chez les individus en fonction de leur appartenance «biologique» au groupe :

Nous sommes tout d'abord issus de parents qui étaient le déroulement d'une race. Et l'on sait que dans le facteur race, il n'y a pas que le sang qui influe : en la race est inscrite l'action des éléments territoriaux, orographiques et climatériques. C'est devenu un cliché poétique que de se proclamer fils du sol, de la montagne, de la mer, des steppes... Les éléments naturels incrustent donc en notre être tout entier des caractères indélébiles dont la somme représente notre individualité. Et comme les mêmes causes produisent les mêmes effets, il s'ensuit que les individus de la même race et du même pays jouissent d'une hérédité commune, possèdent des caractères communs qui deviennent comme le support physiologique du sentiment national (p. 89).

Mais pour des gens comme Lachance, le fait d'avoir une conception de la nation qui comprend un élément de déterminisme ne signifie pas que ce fait soit considéré d'une extrême importance. Lachance lui-même invite d'ailleurs ses lecteurs à saisir la nuance :

Nous rejetons enfin l'opinion [...] de ceux qui accordent au facteur racique ${ }^{19}$ une influence décisive sur la formation du national. La race a sans doute une causalité profonde sur le tempérament humain, mais elle n'est pas d'ordre proprement humain. Elle est un phénomène animal, tandis que la nationalité est un phénomène spécifiquement et exclusivement 
humain. Les animaux sont d'une race; l'homme seul est ressortissant d'une nation (p. 84).

Il prend donc soin de préciser que si la matière influence notre esprit et notre comportement, elle ne règle pas tout, ni sur le plan individuel, ni sur le plan collectif. Ainsi la valeur des individus et des nations n'a pas pour origine la physiologie, dira-t-il, mais l'effort à tendre vers la conquête des valeurs humaines et spirituelles. Le racisme, en préconisant le retour à la pureté matérielle des origines, et surtout en espérant retrouver par ce moyen la pureté spirituelle, préconise donc une philosophie trop matérialiste pour convenir à des esprits libres.

Le racisme descend infiniment [...] bas. En présence du dualisme inhérent à l'être humain, il opte pour la matière. [...] Étendant le déterminisme qui régit l'ordre physiologique au plan de l'activité intellectuelle, morale et artistique, il aboutit à l'hégémonie de la chair et du sang. Il rend le complexe physiologique, la race, quoi!, justiciable des tares et des qualités de l'esprit. [...] Ceux qui se plaisent par un triste travers à tirer avantage des confusions pour mieux fustiger leurs compatriotes et brandir l'anathème sont libres d'appeler ça du nationalisme, nous n'y voyons que du matérialisme cru [...] Cet orgueil de race incommensurable, cette suffisance froide, attentatoire à l'égalité chrétienne, à la justice et la charité, nous répugnent $[\ldots]^{20}$.

Le nationalisme raciste est donc inacceptable pour Lachance, et il refuse que ces accusations soient lancées contre les nationalistes canadiens-français. D'ailleurs c'est le racisme, dit-il, que l'Église a condamné, et non pas le nationalisme comme tel ${ }^{21}$ :

Si la situation actuelle de l'Italie et de l'Allemagne [a forcé les autorités religieuses] à porter de rudes 
coups aux prétentions immodérées de l'orgueil de race, elles ont été constamment soucieuses de manifester leurs sympathies pour l'attachement de l'homme à son pays, à sa mentalité, à ses coutumes, à son histoire (p. 70-71).

De plus, analysant le nationalisme en termes de devoirs de piété, Lachance affirme que :

nous n'avons de devoirs, nous ne sommes liés d'obligations culturelles qu'envers ce qui est supérieur à nous. [Et] si le mot nationalité ne dénonce que combinaison d'éléments chimiques et mécaniques, pourquoi tant de beaux gestes? Enfin, s'il n'y a pas d'idéal en fonction duquel s'établit le prix des choses, comment pouvons-nous conclure à notre valeur et à celle de culture? (p. 129).

Voilà donc une question importante de réglée. Maintenant, d'autres problèmes se posent aux nationalistes, notamment celui de l'équilibre à trouver entre nationalisme et universalisme.

\section{c) La "conversion à l'bumain»}

Un des principaux problèmes que pose la relation entre le nationalisme et le catholicisme se situe dans l'opposition implicite entre particularisme et universalisme. De prime abord, comment en effet ne pas apercevoir l'incompatibilité entre l'insistance du nationalisme sur la singularité de la nation d'une part et, d'autre part, la valeur accordée par le catholicisme à l'universalité de la communauté des croyants? Le nationalisme ne vient-il pas ériger des frontières entre les êtres humains, qui sont pourtant tous frères et sœurs, enfants de Dieu? Cela pose un problème sérieux, auquel Lachance s'attaque d'ailleurs en premier lieu. Il ne s'agit pas d'un faux problème, puisque la critique 
antinationaliste frappe peut-être assez juste : en effet, les nationalistes canadiens-français semblent parfois vouloir s'isoler du monde qui les entoure pour prémunir la nation contre les menaces de son entourage. Mais ce problème, qui peut être compris dans une perspective catholique ${ }^{22}$, Lachance l'aborde d'abord dans une perspective «humaniste».

D'emblée, à ses yeux, il n'existe aucune antinomie entre particularisme et universalisme, entre nationalisme et internationalisme. Ces théories, à son avis, sont des «théories partielles» qui doivent se compléter mutuellement : «Un nationalisme qui ne débouche pas sur un internationalisme judicieux, dit-il, dégénère en chauvinisme ridicule, comme du reste l'internationalisme qui ne repose pas sur le nationalisme perd son nom pour devenir humanitarisme, utopie, chimère de la pire espèce.» (p. 24) Voilà une formule intéressante, mais voyons le raisonnement à sa source.

Selon Lachance, les nations ont un rôle particulier à jouer dans la facilitation de la réalisation des fins de l'homme, qui sont entre autres l'atteinte du bien humain, l'atteinte d'un niveau supérieur de civilisation. Dans cet esprit, la nation est investie d'une mission civilisatrice. Cependant, puisque chaque nation n'est pas en possession de tout le bien humain et qu'elle ne peut espérer y parvenir seule, la collaboration et l'emprunt lui sont nécessaires. Dans le cas canadien-français, par exemple, Lachance affirme que les Canadiens français sont déjà nettement ouverts sur le monde, sur la France catholique surtout, à cause des affinités évidentes entre les deux cultures ${ }^{23}$. Ouverts à l'Italie ensuite, «l'Italie la "claire", la latine, la pittoresque». Mais à côté de ces faits acquis, Lachance constate aussi que certains emprunts pourraient être faits «chez nos voisins» : «Nous pourrions, par exemple [...] recevoir d'eux quelques 
bonnes leçons de pédagogie. La force des choses nous contraint aussi à bénéficier de leur bien-être matériel et à leur demander le secret des succès économiques.» (p. 38). Plutôt que de rejeter en bloc l'influence américaine ou anglo-canadienne, la meilleure attitude serait d'y prendre ce qui manque à la nôtre. Pour Lachance, et même si son ouverture paraît aujourd'hui un peu limitée, les choses sont claires : de la variété naît la richesse et la différenciation de l'humanité en nations est nécessaire pour des raisons de civilisation. L'idée n'est donc pas de les maintenir stérilement dans la pureté de leurs particularités. À cet effet, il lance un avertissement aux nationalistes : "Rêver d'indépendance ${ }^{24}$ et d'isolement, si "superbes" soient-ils, seraient [sid] non seulement égoïsme et orgueil, mais folie et déchéance certaine.» (p. 27). Cette volonté de s'ouvrir au monde, à l'humanité, pour les bénéfices qu'en retirent les cultures et le progrès de la civilisation en général, Lachance l'appelle la nécessaire "conversion à l'humain ${ }^{25}$ ». Cette conversion à l'humain garantit donc qu'au-delà de la conservation des particularismes ethniques, les valeurs humaines universelles ne seront jamais reléguées à l'arrière-plan. Dans le cas des Canadiens français, Lachance juge que cela leur est facile, étant donné qu'à son avis ils sont déjà ouverts à l'extérieur et accueillants. Il croit aussi que

toutes les avanies qu'il a subies n'ont pas laissé de traces : [le Canadien français] n'a gardé aucun préjugé et aucune haine de race. Ses défauts sont plutôt en sens inverse. Ses lacunes sont celles inhérentes à la jeunesse et à la mentalité du vaincu. Il est trop souvent enclin à l'ingratitude et à la susceptibilité. Mésestimant aussi trop facilement ses dons naturels, il est exposé à devenir pessimiste (p. 29).

Enfin, notons que cette conversion à l'humain est aussi, aux yeux de Lachance, un préalable nécessaire à une autre 
conversion, encore plus importante, la «conversion au divin». Mais nous allons trop rapidement, car il nous reste une autre question à examiner avant de passer aux liens entre nationalisme et catholicisme : le lien entre le politique et le national.

\section{d) Le politique et le national}

Pour Lachance l'existence des nations et leur diversité est nécessaire en vue de l'atteinte d'un niveau supérieur de civilisation. Qu'en est-il de l'existence des États? Sa conclusion à ce sujet est très similaire, et c'est ainsi qu'il souligne que «la pluralité des États est également impérieuse» (p. 49) Plus concrètement, Lachance considère que la pluralité des circonscriptions politiques est nécessaire pour préserver l'ordre et éviter l'anarchie. La volonté d'abattre les frontières, dans cette perspective, prend la couleur d'une . idée dangereuse :

c'est une colossale utopie, dit-il, que de rêver enserrer dans un seul réseau politique l'univers tout entier. Le résultat serait forcément l'anarchie. Les circonscriptions politiques sont donc indispensables au règne de l'ordre, à l'épanouissement du bien, au progrès de l'humanité, et par voie indirecte, à l'expansion du christianisme (p. 50).

Lachance remarque que l'État et la nation ont souvent tendance à se confondre. De fait, dit-il, les deux se ressemblent beaucoup. Ce qui les distingue, on l'a déjà aperçu, c'est la nature du lien entre les individus qui les composent : ce qui lie les individus en nations, ce sont les attaches sentimentales; ce qui les lie en un État, ce sont des attaches «juridiques». En d'autre termes, l'unité de la nation se fonde sur l'agrément de vivre ensemble, celle de l'État sur la nécessité de conformer l'action des individus 
aux lois nécessaires au bien commun, de maintenir l'ordre et la justice en somme. Il n'y a donc pas lieu de les identifier outre mesure. À cet effet, Lachance fait d'ailleurs remarquer qu'un régime politique peut être modifié ou remplacé par un autre sans que la nation soit changée pour autant. Il ajoute qu'en plus deux nations peuvent s'associer politiquement «sans pour cela renoncer à leur individualité ethnique» (p. 77), le Canada constituant à cet effet un exemple, bien qu'imparfait il est $\mathrm{vrai}^{26}$.

Par rapport au nationalisme, cette question est d'une certaine importance parce que la confusion entre le politique et le national semble être courante chez les nationalistes $^{27}$. L'accusation de confondre le politique et national était liée à la volonté présumée des nationalistes de vouloir utiliser l'État québécois aux seules fins de la majorité française et catholique ${ }^{28}$. Lachance tente donc de redonner à chacun son rôle propre. On pourrait s'en étonner, mais il semble accorder une valeur plus importante à l'État. C'est que l'ordre politique, dit-il, vise avant tout à poursuivre le bien humain, qui est universel. Le «national», de son côté, vient surtout commander la modalité particulière de cette recherche du bien humain universel. Ainsi, comme le dit Lachance, «'humain conditionne le politique par en-haut, et le national par en-bas.» (p. 83). Ce qui est surtout capital, c'est de ne pas confondre l'un et l'autre : «Une politique qui, au lieu de poursuivre le bien humain, se donnerait comme fin l'exaltation des caractères nationaux et qui, au lieu de se régler sur les principes du droit, se modèlerait sur les aspirations particulières du groupe à gouverner, serait vraiment impropre à servir de liaison à plusieurs entités nationales. Elle ferait la confusion du politique et du national.» (p. 79-80). Quand il analyse cette question en termes de devoirs des membres du groupe envers l'ordre politique 
et l'ordre national, il remarque que de manière générale, le problème ne se pose pas puisque les nations et les États ont tendance à se superposer. Mais dans le cas de l'État canadien, qui recouvre deux nations ${ }^{29}$, les choses sont moins évidentes. Pour solutionner le problème, Lachance conclut que les devoirs envers les deux ordres doivent être distingués en devoirs de justice et de patriotisme. Par devoir de justice, Lachance entend une vertu de volonté «destinée à provoquer et à maintenir l'ordre politique en vue d'assurer la réalisation du bien commun» (p. 186). Par patriotisme, il entend plutôt les devoirs de reconnaissance envers les causes de notre existence. Puisque l'État constitue un «promoteur du bien humain», et que là réside l'objectif essentiel de la communauté, les devoirs de justice jouissent donc a priori dans l'esprit de Lachance d'une "primauté de nature et de fonction sur le patriotisme» (p.187). Conséquemment, les nations qui, pour des raisons diverses, ne pourraient espérer seules aspirer à la réalisation du bien humain, seraient dans l'obligation de se rallier à un seul ordre politique et devraient renoncer à certaines prérogatives pour promouvoir ce bien humain.

Dans le contexte canadien, cela signifie-t-il que les Canadiens français doivent renoncer à quoi que ce soit sur le plan national? Pas tout à fait car au fond, selon Lachance, on ne peut pas dire que la nation canadienne-française doive sa survie (son bien) à l'ordre politique canadien, au contraire (p. 191). Et d'ailleurs, il faut bien remarquer que l'ordre politique au Canada n'est pas constitué que de l'État fédéral, mais aussi des États provinciaux. Dans ce cas, les devoirs de patriotisme des Canadiens français peuvent fort bien aller vers leur nation, et reléguer les devoirs de justice vers les États provinciaux et l'État canadien, dans la mesure où ces derniers ne sont pas source d'injustice et sont 
toujours susceptibles de pourvoir la nation du bien humain. C'est de cette façon que Lachance analyse les rapports entre le politique et le national et qu'il règle la question des devoirs de patriotisme pour les Canadiens français. Le patriotisme s'attache donc à la nation, la justice aux États provinciaux et fédéral. Ces deux devoirs se distinguent, mais ils ne s'opposent pas nécessairement.

La question du sentiment national, du racisme, de l'opposition entre nationalisme et universalisme et entre le politique et le national étant réglées, il nous reste à voir comment Lachance «réconcilie» nationalisme et catholicisme.

\section{e) Nationalisme et catholicisme}

Nous avons vu plus tôt l'idée d'une nécessaire «conversion à l'humain». Pour les raisons que nous avons données, cette conversion est en soi importante pour Lachance, mais elle l'est aussi parce qu'elle constitue un préalable à une conversion encore plus importante : la conversion au $\operatorname{divin}^{30}$.

Lachance rappelle d'abord un fait qui est évidemment pour lui fondamental, soit qu'à l'origine et à la fin de toute chose il y a Dieu. Il va donc de soi que les nations sont ses créations : «Les peuples se différencient entre eux comme les parties de l'univers qu'ils habitent. C'est une nécessité, ajoute-t-il, une donnée de fait. Elle est donc prévue par le Maître de la vie; elle a ses raisons d'être, puisqu'Il a ses vues et ses desseins.» (p. 89) Cette nécessité de fait étant affirmée, il y aurait aussi une "nécessité morale» à l'existence des nations. Lachance la découvre lorsqu'il évalue les modalités de l'épanchement de la grâce dans les âmes. Dieu, qui est soucieux de se modeler aux âmes afin d'y faciliter son infiltration, y parviendrait plus facilement, selon 
Lachance, dans les peuples les «mieux équilibrés et les plus sains» (p. 76). Si on se rappelle le principe voulant que de la variété naisse la richesse et donc que la variété soit nécessaire à l'atteinte plus parfaite du bien humain, on constate que la disparition d'une nation provoquerait un appauvrissement général et une diminution des possibilités d'épanchement de la grâce. Mais Lachance n'insiste pas trop sur cet argument et il s'empresse de préciser que Dieu n'a besoin d'aucune condition particulière pour accomplir son œuvre de rédemption... Au plus, Lachance se permet de conclure que les nations sont utiles à Dieu, mais non nécessaires.

Plus généralement, Lachance énonce aussi au fil des pages quelques principes généraux concernant les rapports entre la foi et l'appartenance nationale, notamment le fait que «Dieu n'a établi aucun rapport nécessaire entre sa loi de grâce et un tempérament ethnique quelconque» (p. 157), et que «l'appartenance à une nationalité n'est ni un moyen de salut, ni un moyen de répulsion» (p. 138), principes qui prennent l'allure de mises en garde pour certains nationalistes qui seraient tentés de lier trop étroitement les deux. Mais d'un autre côté, Lachance peut aussi conclure de ses raisonnements que «l'adhésion à la foi et la naissance à la Vie n'entraînent pas [...] la renonciation [aux] caractères nationaux» (p. 45) et, surtout, qu'il «est en dehors des visées de la grâce d'exterminer les nationalités.» (p. 145) La foi catholique n'entre donc pas en contradiction avec le sentiment national et n'est pas incompatible avec lui.

Mais quels sont les rapports qui doivent s'établir entre le catholicisme et le nationalisme? Nous touchons ici au problème fondamental que tente de résoudre Lachance : comment le sentiment national, ce sentiment puissant «issu de la chair et du sang», doit-il être rationalisé? Cette ques- 
tion est essentielle car «si on soustrait le sentiment national au contrôle actif de la raison pour le lancer dans une arène libre, dit Lachance, il confisque à son profit tous les autres sentiments et dégénère en fanatisme collectif.» (p. 175). Alors comment y arriver? Tout simplement, répond-il, en le remettant à sa place, c'est-à-dire en le gardant au niveau que la raison lui assigne, soit la subordination à la foi catholique.

Rappelons-nous que pour le croyant, la cause première de son existence, de celle de sa famille, de sa nation et ainsi de suite est Dieu. Partant de là, Lachance rappelle que s'il doit y avoir vénération et reconnaissance envers les causes de notre existence, la raison commande aux hommes de les tourner d'abord vers la cause première, ce qui apparait logique. Dans cet ordre, la nation, créée par Dieu, ne se trouve à constituer pour l'homme qu'une cause seconde de son existence. La raison dicte donc que la reconnaissance envers ces deux ordres, leur vénération, ne peut être confondue, ni surtout, ce qui est le plus fondamental, inversée. Lachance rappelle d'ailleurs à ses lecteurs que c'est pour avoir opéré cette inversion des valeurs que la doctrine de Maurras a été condamnée par le Pape : "Charles Maurras, n'étant pas chrétien, a été victime de ce péril. Il a osé subordonner le catholicisme à la civilisation méditerranéenne, le réduisant au niveau inférieur d'instrument de culture.» (p. 63)

De l'avis de Lachance, le clergé n'a pas pour rôle direct de cultiver le patriotisme. Mais rien ne l'empêche de le faire, pourvu bien sûr que soient respectés les quelques principes évoqués plus haut. Il serait même du devoir du clergé de ne pas laisser le sentiment national à lui-même : "C'est donc à l'heure actuelle un devoir pressant de consolider ce sentiment en lui inoculant un ingrédient rationnel, un sur- 
croît d'énergie dirigée, afin de le transformer en habitude vertueuse. Et grâce à ce traitement long et difficile, il s'épanouira en culte subordonné, en religion faite d'attendrissement, de piété et de vénération à l'endroit des causes secondes qui ont tenu auprès de nous la place de Dieu.» (p. 177178). Cette conclusion ne s'applique pas qu'au clergé, mais elle résume bien la proposition générale de Lachance.

Voilà qui résume l'essentiel des idées discutées dans Nationalisme et religion. Nous avons donc vu que l'esprit doit dominer la matière et que le nationalisme, qui est à la base un sentiment, doit être capté par la raison; que le racisme est une doctrine condamnable parce qu'elle donne trop d'importance à la matière face à l'esprit; que la diversité des nations est nécessaire, mais qu'elles ne doivent pas se replier sur elles-mêmes; que même s'ils sont liés, le politique et le national doivent êtres distingués et que les devoirs des individus doivent à leur tour être distingués entre devoirs de patriotisme et de justice; enfin, que la foi catholique n'est pas incompatible avec le national, qu'elle lui est même un corollaire obligé. Ces positions, Lachance les élabore à partir des principes de la foi et de la raison, et c'est cette insistance qu'il met sur la raison qui a fait dire au philosophe Leslie Armour que Louis Lachance a jeté «les bases d'un nationalisme fondé sur la raison, et différent de celui de Lionel Groulx essentiellement fondé sur les sentiments ${ }^{31} »$. Il s'agit là à notre avis d'une simplification presque grossière ${ }^{32}$, mais il n'est pas le seul à avoir remarqué le caractère raisonné du nationalisme de Lachance. Exactement cinquante ans plus tôt, en 1937, un écrivain et journaliste polémiste, réputé pour ses attaques contre les nationalistes et Lionel Groulx en particulier, encensait l'essai de 
Lachance en grande partie pour cette raison : Jean-Charles Harvey.

\section{Un lecteur enthousiaste et inattendu de Lachance : Jean-Charles Harvey}

Bien que son nom évoque surtout le romancier qui a publié en 1934 Les Demi-civilisés ${ }^{33}$, Jean-Charles Harvey a connu une longue carrière journalistique dont l'un des points forts fut la fondation en 1937 de l'hebdomadaire Le Jour et sa publication durant neuf ans. Cet intellectuel au parcours singulier appuya dans un premier temps le nationalisme pour en devenir par la suite un adversaire acharné, eut des sympathies communistes malgré un libéralisme de plus en plus affirmé au cours des années trente, et fut un réformiste qui deviendra davantage conservateur avec le temps, notamment sur la question ouvrière. La fondation du Jour répondait à plusieurs objectifs, dont l'un des principaux était la «lutte contre le cri de race et contre toute forme de nationalisme démagogique» et «l'opposition nette et énergique au séparatisme $[. . .]^{34} \%$. En fait, Harvey fut l'un des critiques du nationalisme les plus actifs au cours des années trente ${ }^{35}$.

Quand il s'attaquait aux nationalistes canadiens-français, quatre critiques revenaient particulièrement souvent sous sa plume. Nous les présentons ici en ordre croissant d'importance, si la plus grande récurrence d'une idée sous la plume d'un intellectuel constitue une indication relativement sûre de l'importance qu'il lui accorde ${ }^{36}$. La première est que les nationalistes refuseraient de reconnaître que l'individu est plus important que la nation, et que ce qui constitue le caractère universel du genre humain est plus important que les différences entre les groupes qui le composent. La deuxième est que le nationalisme en général, mais 
particulièrement le nationalisme canadien-français, constitue une attitude passéiste et antiprogressiste. La troisième est que le nationalisme serait la plupart du temps associé à la haine, au racisme et à violence. La quatrième, enfin, est que les nationalistes ne se laissent guider que par les sentiments et non par la raison. Parce qu'il critique essentiellement ces mêmes «excès» du nationalisme, et principalement l'excès de sentiment et le manque de raison, Lachance aura l'appui sans réserve d'Harvey.

C'est en 1937, dans un article intitulé «Nationalisme passionnel et nationalisme humain ${ }^{37}$ » qu'Harvey rédige une critique élogieuse de Nationalisme et religion, uun des livres les plus intelligents que l'on ait écrit sur la question», à son avis $^{38}$. Un fait intéressant à remarquer dans ce texte est qu'on y retrouve évoqués trois des principaux thèmes de sa critique du nationalisme. Il n'y manque, à vrai dire, que la question du passéisme et de l'antiprogressisme, mais Lachance n'insiste pas particulièrement sur l'importance du passé et des traditions dans son ouvrage ${ }^{39}$. On retrouve donc l'idée de la prédominance des valeurs humaines et universelles sur les intérêts nationaux, la dénonciation du racisme et l'importance de la raison. À cela, ajoutons la nécessité de distinguer le politique du national.

On pourra peut-être s'en étonner, mais si l'argumentation de Lachance est fondée sur la raison, Harvey ne semble pas se soucier du fait qu'elle l'est avant tout sur la foi. Si l'on exclut la possibilité de l'existence de Dieu, pourtant, une bonne partie de l'argumentation de Lachance tombe à l'eau et Harvey lui-même n'est pas croyant. Du moins c'est l'aveu qu'il faisait à Groulx dans une lettre en novembre $1935^{40}$. Par ailleurs, s'il n'avait pas la foi et s'il critiquait souvent la trop grande place du clergé dans la société (dans l'éducation particulièrement), il n'était pas un anticlérical 
radical. C'est ainsi avec beaucoup de respect qu'il pouvait parler de certains membres du clergé faisant figure d'intellectuels, dont le frère Marie-Victorin. Lachance, à son tour, ne lui inspirera pas autre chose que de l'admiration. Voyons ce qu'il a retenu de sa pensée.

Quand Harvey discute de la question de l'opposition entre l'individu et la nation, entre l'«humain» et le «nationab, on remarque que sa position au sujet du nationalisme est assez nuancée, tant que ce dernier n'entre pas en opposition avec les valeurs humaines :

Certes, dit-il, tout homme de cœur doit défendre son individualité dans la mesure où la société n'en souffre pas, comme il doit défendre sa famille dans tous ses droits légitimes; et la nation n'étant que la famille agrandie, il doit même la défendre contre tout empiétement qui pourrait l'humilier, lui ravir des habitudes chères, des traditions, du bien-être, une langue, une foi. Il doit défendre cela jalousement, mais en respectant toujours l'ordre humain et universel dans les bornes de la Loi suprême qui est au-dessus de tout et pour laquelle les nations ne sont que de splendides accidents ${ }^{41}$.

Harvey remarque aussi positivement le fait que Lachance «approuve le nationalisme dans la mesure où il ne vient pas en conflit avec les lois essentielles qui forment ce que j'appellerais le droit humain, qui est un droit éternel et universe ${ }^{42} . » C e$ qu'il ne mentionne pas, c'est qu'en fait, dans l'esprit de Lachance, c'est d'abord et avant tout la subordination à la foi catholique qui lui permet réellement d'approuver le nationalisme. Sans déformer la pensée de Lachance, Harvey n'y prend néanmoins que ce qui lui convient. 
La condamnation du racisme par Lachance constitue un autre facteur d'approbation pour Harvey. Puisque les deux hommes partagent une conception similaire de la nation, c'est-à-dire une conception culturelle mêlée d'une part de prédisposition psychologique liée à l'héréditét ${ }^{43}$, on ne s'étonnera pas du fait qu'il ne critique pas cette opinion, mais qu'à l'instar du dominicain il condamne néanmoins le racisme, qui est une exagération de la valeur des traits physiologiques et de leur impact sur la valeur de la nation elle-même. Pour Harvey, le racisme, c'est :

[...] la suprême abomination. [...] Les racistes peuvent fort bien s'assimiler à des troupeaux de grands animaux pur sang, dit-il, plus disciplinés sans doute que les meutes de loups, mais non moins sanguinaires et voraces [...] Le racisme, c'est une philosophie diabolique et c'est cette philosophie qu'admirent quelques-unes de nos bonnes âmes ${ }^{44}$.

Nul besoin de s'étendre plus longuement là-dessus : Harvey approuve totalement l'antiracisme de Lachance.

Harvey juge aussi positivement la distinction qu'impose le philosophe entre le politique et le national parce qu'à son avis, la confusion de ces deux ordres sert de justification aux actions des pires régimes politiques :

Pour comble d'horreur, dit-il, on a fusionné la notion d'État et la notion de patrie, de façon à mieux soumettre les peuples à des formules ou régimes politiques qui sacrifient l'humain, le rationnel, je dirais le bonheur des individus, à ce qu'il y a de plus précaire, de plus conventionnel et de plus arbitraire dans l'existence d'une agglomération humaine ${ }^{45}$.

Ailleurs il écrit, à la suite de Lachance, "[s]'il faut considérer toutes les questions politiques uniquement dans leur rapport avec l'intérêt national, on justifie par le fait toutes 
les spoliations, tous les coups de force ${ }^{46} . »$ Il ne le mentionne pas dans ce texte, mais on pourrait croire que le séparatisme, qui est ni plus ni moins que l'application du principe voulant que État et nation doivent se superposer exactement, est une autre raison qui le fait applaudir à cette distinction de Lachance ${ }^{47}$.

Enfin, il ne fait pas de doute qu'un des principaux facteurs, sinon le facteur principal, qui permet à Harvey de se montrer d'accord avec le nationalisme tel que le conçoit Lachance est l'aspect rationnel du discours de ce dernier et l'importance qu'il accorde à la raison en général. Harvey voit Nationalisme et religion comme un des livres les plus intelligents» que l'on ait écrits sur le nationalisme. Son auteur? Un religieux qui «a parfaitement vu, comme tous les véritables intellectuels et penseurs de notre époque, jusqu'à quel point le nationalisme moderne s'est fourvoyé», et dont le livre offre un "raisonnement [qui] forme une chaîne continue ${ }^{48}$ \%. L'analyse d'Harvey, qui se transforme en réquisitoire contre certaines formes de nationalisme, est parsemée de références à la «raison simple et nue», au «bon nationalisme [...] qui s'appuie sur les forces de l'esprit», à «la pensée qui pense et [...] la raison qui raisonne» et au fait que "plus un individu s'élève, que son cœur s'élargit, que son intelligence s'éclaire, que sa raison s'équilibre, il se dépouille d'une foule d'éléments passionnels qui entravaient ses facultés supérieures», etc. Peut-être Harvey aurait-il révisé son jugement s'il avait pris conscience alors du fait que Lachance pouvait prononcer des discours patriotiques qui faisaient appel au sentiment tout en prônant, comme philosophe, un nationalisme très rationnel. Mais il est déjà extraordinaire que le fondateur du Jour ait pris le temps de lire cet ouvrage d'un religieux nationaliste, et surtout que 
Lachance ait réussi à satisfaire les exigences d'un des plus virulents critiques du nationalisme canadien-français.

\section{$* * *$}

Lachance a apporté une contribution intéressante à la réflexion philosophique et théologique sur le nationalisme. À ce titre, il semble bien qu'il constitue une exception. Selon Gregory Baum, en effet, peu nombreux sont les représentants de l'Église qui ont été soucieux d'élaborer à l'usage des nationalistes catholiques une doctrine pour guider leur action. C'est ainsi qu'il exprime sa surprise devant le peu d'intérêt de l'Église pour le nationalisme :

La théologie systématique et l'éthique ont très peu réfléchi au phénomène polymorphe du nationalisme. [...] l'enseignement social catholique, source documentaire importante et abondante en matière d'éthique sociale et économique, ne propose aucun traitement systématique du nationalisme. Le nationalisme est à peine mentionné dans les encycliques papales sur les questions sociales ${ }^{49}$.

Dans Le nationalisme : perspectives éthiques et religieuses, Baum ne parle pas du Nationalisme et religion de Lachance, qui constitue pourtant un excellent exemple de réflexion philosophique et théologique catholique sur le nationalisme. Baum s'attarde plutôt à rapporter la pensée du philosophe québécois Jacques Grand'Maison dans un livre qu'il avait justement intitulé, trente-quatre ans après Lachance... Nationalisme et religion ${ }^{50}$.

\section{NOTES}

${ }^{1}$ Voici, en ordre chronologique, les principaux ouvrages publiés depuis les trente dernières années sur la philosophie au Québec : Leslie 
Armour et al., Essais de philosophie politique québécoise, (XVIII-XIX siècles), Université du Québec à Montréal, 1992, 113 p.; Jacques Beaudry, Autour de Jacques Lavigne, philosophe : histoire de la vie intellectuelle d'un philosophe québécois de 1935 à aujourd'bui, Trois-Rivières, Éditions du Bien public, 1985, 168 p.; Jacques Beaudry, La Philosophie et le Québec: des noms et des notes, Sherbrooke, Ex libris, 1988; Société de philosophie du Québec, Philosophie au Québec, Montréal, Bellarmin, 1976, 263 p.; Marc Chabot et Jacques G. Ruelland, Figures de la philosophie québécoise à l'époque de la Révolution française, Montréal, UQAM, 1989, 2 v.; Roland Houde, Histoire et philosophie au Québec : anarchéologie du savoir historique; Trois-Rivières, Éditions du Bien public, 1979, 183 p.; Institut supérieur des sciences humaines, Matériaux pour l'bistoire des institutions universitaires de philosophie au Québec, Québec, ISSH (Université Laval), 1976, 2 v.; Raymond Klibansky et Josianne BouladAyoub, La pensée philosophique d'expression française au Canada : le rayonnement du Québec, Sainte-Foy, Presses de l'Université Laval, 1998, 686 p.; Yvan Lamonde, La philosophie et son enseignement au Québec (1665-1920), LaSalle, Hurtubise HMH, 1980, 312 p.

${ }^{2}$ Louis Lachance, Saint Thomas dans l'bistoire de la logique, extrait des Études d'histoire doctrinale et littéraire du XII ${ }^{e}$ siècle, Ottawa/Paris, Institut d'études médiévales/Vrin, 1932, 45 p.; Le concept de droit selon Aristote et saint Thomas, Montréal/Paris, Éditons Albert Lévesque/ Librairie du Recueil Sirey, 1933,442 p.; Où vont nos vies?, Montréal, L'Euvre de Presse dominicaine, 1934, 216 p.; Nationalisme et religion, Ottawa, Collège dominicain, 1936, 195 p. et enfin L'bumanisme politique de saint Thomas - Individu et État, Ottawa/Paris, Éditions du Lévrier/Librairie du Recueil Sirey, 1939, 2 vol. (maîtrise en théologie).

${ }^{3}$ Groulx semble avoir apprécié l'ouvrage de Lachance. En effet, dans une causerie prononcée en septembre 1936 il déclarait : «Énonçons un principe doctrinal que j'emprunte au livre lumineux du Père Louis Lachance, o.p., Nationalisme et religion [...]», L. Groulx, "Labeurs de demain ", dans Directives, Montréal, Éditions du Zodiaque, 1937, p. 128. Albert Lévesque, qui lançait en 1934 son ouvrage intitulé La nation canadienne-française. Son existence, ses droits, ses devoirs, témoignait aussi de cette visibilité croissante : «Pour rédiger ce lexique, nous avons eu recours, au préalable, aux lumières et à la générosité d'un jeune philosophe, dont la réputation grandit rapidement, le R. Père Louis Lachance, O.P., auteur du Concept de droit selon Aristote et S. Thomass. 
On notera que c'est le même Lévesque, à titre d'éditeur, qui avait publié cet ouvrage un an auparavant.

${ }^{4}$ Louis Lachance, Philosophie du langage, Ottawa, éditions du Lévrier, 1943, $216 \mathrm{p}$.

${ }^{5}$ Soit L'être et ses propriétés, Montréal, Éditions du Lévrier, 1950, 234 p., La lumière de l'âme. Vingt entretiens sur la grâce, Montréal, Éditions du Lévrier, 1954, 224 p. et Le droit et les droits de l'homme, Paris, Presses universitaires de France, 1959, 238 p.

${ }^{6}$ Pour une description plus détaillée et plus sensible des principaux événements de la vie de Lachance, on consultera la notice nécrologique rédigée par le Frère Antonin Papillon, o.p., quelques semaines après la mort du Père Lachance et publiée dans Antonin-M. Plourde, Qui sont-ils et d'où viennent-ils? Nécrologue dominicain 1882-1964, Montréal, Éditions du Lévrier, 1964, pp. 1-16.

${ }^{7}$ Étonnamment, R. Hamel, J. Hare et P. Wyczynski, directeurs du Dictionnaire des auteurs de langue française en Amérique du Nord, Montréal, Fides, 1989, xxvi-1364 p., n'ont pas jugé bon de lui accorder une notice.

${ }^{8}$ Nous reprenons ici et dans le prochain paragraphe les formulations de Jean-Claude Dupuis dans son article «Nationalisme, séparatisme et catholicisme dans l'entre-deux-guerres», dans Jocelyn Saint-Pierre et Michel Sarra-Bournet, Les nationalismes au Québec du $X I X^{c}$ au $X X^{c}$ siècle, Sainte-Foy, Presses de l'Université Laval, 2001, pp. 95-104.

${ }^{9}$ Propos issus du Rapport Dumont rapportés par Guy Bourgeault, «Le nationalisme québécois et l'Église», Revue canadienne des études sur le nationalisme, vol. $5, \mathrm{n}^{\circ} 1$ (printemps 1978), p. 188.

${ }^{10}$ Il s'agit du débat autour de la formule «La langue, gardienne de la foì.

${ }^{11}$ En effet, dans ses Mémoires, Groulx laisse entendre que cela ne l'avait pas inquiété. Pour le prouver, il rapporte ces propos, qu'il aurait tenus à Omer Héroux à l'époque : «Je l'avoue en toute candeur, cette encyclique ne m'inquiète nullement. Après tout le Saint-Père ne peut rien changer à la théologie ni à la philosophie. Il ne peut contester aux petits peuples leur droit à la vie, surtout dans le cas d'un petit peuple comme le nôtre qui fonde sa volonté de vivre, non point sur le seul droit naturel et historique, ni encore moins sur le nouveau droit des 
peuples à disposer d'eux-mêmes, mais sur un droit positif et constitutionnel progressivement accru depuis la Conquête, c'est-à-dire depuis 150 ans», L. Groulx, Mes Mémoires, tome 2 (1920-1928), Montréal, Fides, 1971 , p. 227. Notons que Groulx faisait référence à un projet d'encyclique dirigé contre le régime de Mussolini et le nationalisme en général qu'aurait préparé Pie XI, encyclique qui ne vit finalement jamais le jour. En 1953, dans une conférence intitulée «Catholicisme et action nationale», dans Pour bâtir, Montréal, L'Action nationale, 1953, p. 150, il se proposait de répondre à la question suivante : «Au Canada français, un jeune catholique peut-il, en toute sécurité de doctrine, s'adonner à l'action nationale, c'est-à-dire aimer, servir sa nationalité, sa culture, sa civilisation, et au besoin les défendre?»

${ }^{12}$ Voir Damien-Claude Bélanger, "L'abbé Lionel Groulx et la crise sentinelliste ", dans Mens, vol. 1, $\mathrm{n}^{\circ} 1$ (automne 2000), pp. 7-36.

${ }^{13}$ Lachance, Nationalisme et religion, op. cit., p. 13. Pour alléger le texte, toutes les citations issues de cet ouvrage seront dorénavant indiquées par une simple mention du numéro de page entre parenthèses dans le texte.

${ }^{14}$ Louis-Adolphe Pâquet (1859-1941), prêtre; professeur à la Faculté de théologie de l'Université Laval (1883-1942), doyen de cette même faculté (1904-1938); protonotaire apostolique (1902); vicaire général du diocèse de Québec (1909); directeur et président de l'École supérieure de philosophie, la future Faculté de philosophie de l'Université Laval (1926-1935); fondateur de l'Académie canadienne Saint-Thomas d'Aquin (1930). Yvan Lamonde, Louis-Adolphe Pâquet, Montréal, Fides, 1972, 87 p. (Coll. «Classiques canadiens»).

${ }^{15}$ Alors que ce qui lie l'État, c'est le droit, des attaches juridiques.

${ }^{16}$ Lachance, Philosophie du langage, op. cit.

17 «[...]comme toute entité distincte est pourvue par la nature de l'instinct de conservation, il est normal que cette conscience de son individualité provoque chez la nation un vouloir-vivre commun». Ibid., p. 109.

${ }^{18}$ Lachance, Nationalisme et religion, pp. 56-57. Nous devons faire remarquer que malgré cette distinction établie, Lachance n'est pas constant dans l'utilisation de l'un ou l'autre. Il utilise ainsi souvent le terme «nationalisme» alors qu'il devrait utiliser le terme "patriotisme». 
${ }^{19}$ Cet adjectif, qui n'est plus utilisé aujourd'hui, pouvait signifier aussi bien «racial» que «raciste». C'est dans sa première acception que Lachance l'utilise ici.

${ }^{20}$ Lachance, Nationalisme et religion, op. cit., pp. 64-66. Cette dernière citation, on l'aura peut-être remarqué, indique clairement que des accusations faisant un lien entre le nationalisme, le racisme et une attitude anti-chrétienne, étaient lancées aux nationalistes.

${ }^{21}$ D'ailleurs, sans en faire un argument, il constate que le Saint-Siège n'a jamais mentionné le terme «nationalismus» dans ses écrits. Ibid., p. 69.

${ }^{22}$ Précisons qu'il existe une critique antinationaliste marxiste, opposant l'internationalisme au nationalisme, vu comme une idéologie bourgeoise ne servant qu'à masquer les enjeux et l'universalité de la condition prolétarienne et aussi, bien souvent, une critique antinationaliste libérale, mettant l'accent sur l'importance de l'individu face à la nation, et débouchant sur une sorte d'internationalisme.

${ }^{23}$ Lachance, Nationalisme et religion, op. cit., p. 38. «Ses produits, dit-il, constituent un aliment apprêté pour nous, immédiatement assimilable, approprié à nos goûts et à nos besoins.»

${ }^{24}$ Lachance utilise certainement ici le terme «indépendance» au sens d'autarcie.

${ }^{25}$ On se rappellera que Groulx et Harvey ont tous deux utilisé cette expression : l'ont-ils trouvée chez Lachance? Ou était-elle seulement dans l'air du temps?

${ }^{26}$ «ll va sans dire que notre unité politique, si elle était comprise, si elle n'était pas faussée par l'ostracisme pratique de l'anglo-protestant, serait parfaitement conciliable avec la liberté nationale.», Lachance, $\mathrm{Na}$ tionalisme et religion, op. cit., p. 78.

${ }^{27}$ C'est ce que suggère l'intérêt que porte Lachance à cette question, à laquelle il accorde dix pages.

${ }^{28}$ Groulx, par exemple, a déjà exprimé une idée semblable : «Quand nous parlons, en effet, d'État français, nous n'exigeons nul bouleversement constitutionnel. [...] Nous demandons seulement de faire [...] un État qui gouverne d'abord pour les nationaux de cette province, pour la majorité de la population qui est canadienne-française.» Lionel Groulx, Leçons professées à l'«École de Vaudreuil), 15 août 1937. Ar- 
chives du Centre de recherche Lionel-Groulx (ACRLG), Fonds LionelGroulx, P1, Ma-243, [p. 36].

${ }^{29}$ Et même davantage, mais l'époque veut que les nations amérindiennes ne soient pas encore vraiment reconnues comme telles.

${ }^{30}$ «La conversion à l'humain est une condition préalable à celle au divin [car] en fermant son cœur au premier on risque d'empêcher toute infiltration du second». Ibid., p. 28.

${ }^{31}$ Leslie Armour, "Louis Lachance», L'Encyclopédie du Canada, Montréal, Stanké, 1987 [1985], tome II, p. 1064. Dans l'édition 2000, Armour écrit maintenant : «L'ouvrage de Lachance Nationalisme et religion (1936) sert de base au nationalisme fondé sur la raison, par opposition au nationalisme préconisé par Lionel Groulx, qui s'appuie surtout sur l'émotion», L. Armour, "Louis Lachance», dans L'Encyclopédie du Canada 2000, Montréal/Paris/New-York, Stanké, 2000, p. 1364.

${ }^{32}$ À notre avis, Lachance et Groulx partageait un nationalisme tout à fait similaire. À cette époque d'ailleurs, Lachance, qui était de vingt-etun ans son cadet, considérait Groulx comme un maître sur la question nationale. En retour, Groulx avait été impressionnée par l'ouvrage de Lachance et lui a demandé conseil à quelques reprises (voir l'édition critique d'une lettre de Lachance à Lionel Groulx que nous avions présentée dans Mens, vol. 1, n 2 (printemps 2001), pp. 163-169). Par ailleurs, des textes de conférences patriotiques qu'il a données révèlent un style oratoire très proche de celui de Groulx, dont une grande partie des textes étaient en fait des transcriptions écrites de conférences. Il faut croire qu'Armour ne les a pas consultés.

${ }^{33} \mathrm{Ce}$ roman, très critique envers la bourgeoisie locale et jugé dangereux pour les bonnes mours de la population, fut aussitôt condamné par le cardinal Villeneuve, archevêque de Québec. Cela coûta à Harvey son poste de rédacteur en chef du Soleil qu'il occupait depuis 1927. Le gouvernement libéral de Taschereau ne pouvait se permettre de garder aux commandes de l'organe officiel du parti un journaliste aussi «dangereux» pour le maintien des bonnes relations entre le gouvernement et l'épiscopat catholique de la province.

${ }^{34}$ Jean-Charles Harvey, «Notre programme», Le Jour, 6 novembre 1937, p. 8. Ce programme (dont une version précédente et légèrement différente fut distribuée au public en guise de présentation du projet de journal) contenait dix points. 
${ }^{35}$ C'est un avis partagé par un des biographes de Harvey, Marcel-Aimé Gagnon, qui note à ce sujet "qu'aucun journaliste, aucun écrivain n'a lutté avec autant d'ardeur et de ténacité que Jean-Charles Harvey contre le séparatisme ou toute autre forme de nationalisme». Marcel-Aimé Gagnon, Jean-Charles Harvey, précurseur de la Révolution tranquille, Montréal, Beauchemin, 1970, p. 158.

${ }^{36}$ Nous sommes arrivé à cette conclusion après avoir analysé quatrevingt deux articles d'Harvey publiés dans Le Jour entre le 16 septembre 1937 et le 31 décembre 1939 qui avaient le nationalisme comme sujet principal. Voir Yves Bégin, Raison et sentiment. Nationalisme et antinationalisme dans le Québec des années 1935-1939. Mémoire de maîtrise (Histoire), Université de Montréal, 2001, 135 pages.

${ }^{37}$ Jean-Charles Harvey, «Nationalisme passionnel et nationalisme humain", dans Art et combat, Montréal, Éditions de l'Action canadiennefrançaise, 1937, p. 212.

${ }^{38}$ Ibid., pp. 212.

${ }^{39}$ Mais cela ne veut pas dire que Lachance n'y tient pas. Ainsi, dans le manuscrit d'une conférence intitulée "Les valeurs nationales», il consacre plusieurs pages à la présentation et à l'analyse des différentes traditions nationales canadiennes-françaises. Lachance recherche dans ce texte les valeurs qui forment l'essence de la nationalité canadiennefrançaise. Il les distingue en deux groupes : les valeurs maitresses (culture française et foi catholique) et les valeurs auxiliaires (les traditions et les lois). Louis Lachance, "Les valeurs nationales», manuscrit dactylographié, Archives des Dominicains ou Frères prêcheurs du Canada, Fonds Louis-Lachance, F1, C7, 158, 10, 23 pages.

${ }^{40} \mathrm{Il}$ avouait à Groulx que ce qui les séparait, ce sont «les trois lettres du mot Foì. Lettre de Jean-Charles Harvey à Lionel Groulx, Québec, 29 novembre 1935, ACRLG, Fonds Lionel-Groulx, P1/A, 1730.

${ }^{41}$ Harvey, "Nationalisme passionnel et nationalisme humain», loc. cit., p. 216. Doit-on voir dans cette évocation de la «Loi suprême» une loi d'ordre naturel ou surnaturel (Dieu)?

${ }^{42}$ Ibid., p. 214.

${ }^{43}$ Ibid., p. 217 : «Que la naissance, l'bérédité, la langue, le climat, l'éducation et le milieu m'aient donné un caractère spécifique dans la grande 
famille humaine, ce caractère j'y tiens et je veux bien le conserver [...]». Nous soulignons.

${ }^{44}$ Ibid., p. 215.

${ }^{45}$ Ibid., p. 211. Rappelons que pour Harvey, les nations sont des «faits purement accidentels et géographiques».

${ }^{46}$ Ibid., p. 214.

${ }^{47} \mathrm{La}$ tendance vers le séparatisme, en fait, pourrait être ajoutée à la liste des griefs d'Harvey contre le nationalisme, mais elle ne revenait pas aussi souvent que les autres dans les années étudiées. En 1962, à soixante et onze ans, il a écrit un livre intitulé : Pourquoi je suis antiséparatiste, Montréal, Éditions de l'Homme, Ottawa, 1962, 123 p., une réponse au livre de Marcel Chaput, Pourquoi je suis séparatiste, Montréal, Éditions du Jour, 1961, $156 \mathrm{p}$.

${ }^{48}$ Ibid., p. 212.

49 Gregory Baum, Le nationalisme : perspectives éthiques et religieuses [trad. de l'anglais], Saint-Laurent, Bellarmin, 1998, p. 16.

50 Jacques Grand'Maison, Nationalisme et religion, Montréal, Beauchemin, 1970, 2 v. 